\title{
Acute Tumor Lysis Syndrome during Oral Fludarabine Treatment for CLL - a Rare Event that Might Be Observed More Frequently in the Future
}

\author{
Catherine Rioufol ${ }^{a} \quad$ Bertrand Coiffier ${ }^{b}$ \\ a Pharmacy, Centre Hospitalier Lyon-Sud, \\ ${ }^{b}$ Department of Hematology, Centre Hospitalier Lyon-Sud and Université Claude Bernard, Lyon, France
}

Tumor lysis syndrome (TLS) with acute renal failure is a very rare complication of oral fludarabine therapy in patients with chronic lymphocytic leukemia (CLL). Calvo-Villas et al. [1] report in this issue of ONKOLOGIE the second published case of such a complication, alerting clinicians to this potentially lifethreatening syndrome that may occur in patients with CLL after fludarabine given intravenously or orally. Treatment with rasburicase and hydration is recommended in front of such a patient but prophylactic measures for patients with high risk of TLS are easy and should be done in all patients.

Fludarabine is a frequently used purine analogue in the treatment of patients with CLL. It has been demonstrated to be superior to regimens based on alkylating agents in inducing clinical and hematologic remissions in both treated and previously untreated patients. Today it is mostly used in regimens like FC (fludarabine plus cyclophosphamide) or FCR (fludarabine, cyclophosphamide, and rituximab). Fludarabine alone is usually prescribed in elderly patients where these regimens might be too toxic. Although TLS and its life-threatening complication, acute renal failure, is a well-known safety problem, reports of TLS in CLL patients treated with fludarabine remain anecdotal, probably because this side effect is easily prevented. Unlike TLS occurring in lymphoma patients treated with chemotherapy, TLS after fludarabine in CLL patients usually occurs with some delay after the first day of treatment, and may be observed up to 7 days after treatment. It only occurs in patients with high a number of lymphocytes in the blood, however, no threshold was defined, or in patients with high tumor mass during the first cycle of therapy. It is easily prevented with administration of allopurinol from day 1 to 10 of the first cycle in high-risk patients. Finally, this event is specific for CLL patients and was not described in other lymphoproliferative disorders where fludarabine may be used, such as follicular lymphoma, mantle cell lymphoma or Waldenström's macroglobulinemia. It is probably a rare event:
Cheson reviewed 6,137 patients with CLL treated with fludarabine and found an incidence of TLS in $0.33 \%$ of the patients [2]. TLS has also been observed in CLL patients treated with oblimersen, flavopiridol, high-dose corticosteroids, and rituximab [3-6].

TLS is caused by the lysis of malignant cells by cytotoxic chemotherapy, leading to a rapid release of intracellular contents into the blood stream including potassium, phosphate, and purines from the breakdown of nuclear proteins. Secondary hypocalcemia, hyperkalemia and hyperphosphatemia lead to gastrointestinal disturbances, neuromuscular effects and cardiovascular consequences. Purines are catabolized to hypoxanthine, then xanthine, and finally in the liver to uric acid by the enzyme xanthine oxidase. The precipitation of excess uric acid contributes to impaired renal function; acute renal failure represents the most frequent and serious clinical consequence of TLS-related hyperuricemia [7]. Risk factors include the type of malignancy, some tumor-related factors, such as high numbers of circulating tumor cells and a large tumor burden, the presence of individual factors, such as preexisting renal insufficiency, and the type and intensity of anticancer regimen used [7].

A literature survey in 2003 reported 5 cases of intravenous fludarabine-induced TLS in patients with CLL that occurred within the first week of initiation of therapy [8]. However, the 20 cases reported by Cheson in 1998 were not accounted for in this survey [2]. Since then some other case reports have been published but they are rare because most of the patients at risk received allopurinol prophylaxis. It is probable that the incidence is higher, most cases not being reported anymore. Since allopurinol prophylaxis is available for oral use in Europe, only one case of oral fludarabine-induced TLS was described [9]. The patient, which had been treated with oral fludarabine $40 \mathrm{mg} / \mathrm{m}^{2}$ daily for 5 days, developed 2 episodes of acute TLS 17 and 15 days after starting fludarabine for the

\begin{tabular}{ll}
\hline KARGER & @ 2008 S. Karger GmbH, Freiburg \\
$\begin{array}{l}\text { Fax +49761 4520714 } \\
\begin{array}{l}\text { E-mail Information@Karger.de } \\
\text { www.karger.com }\end{array}\end{array}$ & $\begin{array}{l}\text { Accessible online at: } \\
\text { www.karger.com/onk }\end{array}$ \\
\end{tabular}


first and second treatment. The authors concluded that fludarabine appeared to cause TLS later after oral administration compared to intravenous therapy. In the case reported in this issue, TLS occurred at day 7.

The standard management strategy for treating TLS is based on hydration and decreasing uric acid concentration. This may be obtained with rasburicase, a newly developed recombinant urate oxidase, that converts uric acid to allantoin, a much more soluble product $[10,11]$. Several studies have demonstrated that rasburicase is generally well-tolerated, although severe adverse effects were reported such as hypersensitivity reactions [10]. Allopurinol has a great efficacy for preventing TLS in high-risk patients but has a limited activity when TLS is installed and creatinine levels have increased.

It has been suggested that CLL patients should receive allopurinol routinely during the first course of fludarabine therapy, however, we think that it has only a role in high-risk patients [10]. These patients may be defined by a high blood lymphocyte count (greater than $100 \times 10^{6} \mathrm{G} / \mathrm{l}$ ) or high tumor mass (multiple lymph nodes larger than $5 \mathrm{~cm}$ ). In a high-risk patient allopurinol should be given for 10 days because TLS is often delayed with fludarabine. In case of oral fludarabine, 15-20 days might be better because 2 of the 3 reported incidences occurred after 15 days.

Calvo-Villas's paper confirms the strong clinical benefits of the newly developed recombinant urate oxidase rasburicase, to achieve a complete recovery of renal function and a decrease of the uric acid level to within the normal range in patients with acute renal failure secondary to TLS. The short 2-days treatment was sufficient but sometimes 3-7 days are necessary to normalize uric acid and creatinine levels. In the case of CLL and fludarabine where TLS occurs later than with chemotherapy in lymphomas, a short treatment is usually sufficient [12]. Furthermore, patients themselves should be educated about the symptoms associated with TLS and the need for high fluid intake during the first course of treatment.

In conclusion, TLS is a rare event in CLL patients but one that may occur more frequently because of the very active armamentarium that is currently developed for these patients. It usually occurs 7-10 days after treatment. It is easily prevented with allopurinol and must be treated with hydration plus rasburicase.

\section{References}

1 Calvo-Villas JM, Martín Urcuyo B, Morales Umpierrez A, Sicilia F: Acute tumor lysis syndrome during oral fludarabine treatment for chronic lymphocytic leukemia. Role of treatment with rasburicase. Onkologie 2008;31 DOI: 10.1159/000118524.

$\checkmark 2$ Cheson BD, Frame JN, Vena D, Quashu N, Sorensen JM: Tumor lysis syndrome - an uncommon complication of fludarabine therapy of chronic lymphocytic leukemia. J Clin Oncol 1998;16: 2313-20.

3 O'Brien S, Moore JO, Boyd TE, et al.: Randomized phase III trial of fludarabine plus cyclophosphamide with or without oblimersen sodium (Bcl-2 antisense) in patients with relapsed or refractory chronic lymphocytic leukemia. J Clin Oncol 2007; 25:1114-20.
4 Byrd JC, Lin TS, Dalton JT, et al.: Flavopiridol administered using a pharmacologically derived schedule is associated with marked clinical efficacy in refractory, genetically high-risk chronic lymphocytic leukemia. Blood 2007;109:399-404.

5 Vaisban E, Zaina A, Braester A, Manaster J, Horn Y: Acute tumor lysis syndrome induced by highdose corticosteroids in a patient with chronic lymphatic leukemia. Ann Hematol 2001;80:314-5.

6 Yang HH, Rosove MH, Figlin RA: Tumor lysis syndrome occurring after the administration of rituximab in lymphoproliferative disorders: High-grade non-Hodgkin's lymphoma and chronic lymphocytic leukemia. American J Hematol 1999;62:247-50.

7 Michallet AS, Tartas S, Coiffier B: Optimizing management of tumor lysis syndrome in adults with hematologic malignancies. Support Cancer Ther 2005;2:159-66.
8 Hussain K, Mazza JJ, Clouse LH: Tumor lysis syndrome (TLS) following fludarabine therapy for chronic lymphocytic leukemia (CLL): Case report and review of the literature. Am J Hematol 2003; 72:212-5.

9 Ramachandran A, Majumdar G: Acute tumour lysis syndrome after oral fludarabine in a patient with chronic lymphocytic leukaemia. Hematol J 2004;5:528-9.

10 Coiffier B, Mounier N, Bologna S, et al.: Efficacy and safety of rasburicase (recombinant urate oxidase) for the prevention and treatment of Hyperuricemia during induction chemotherapy of aggressive non-Hodgkin's lymphoma: Results of the GRAAL1 study. J Clin Oncol 2003;21:4402-6.

11 Hochberg J, Cairo MS: Tumor lysis syndrome: current perspective. Haematologia 2008;93:9-13.

12 Hutcherson DA, Gammon DC, Bhatt MS, Faneuf $\mathrm{M}$ : Reduced-dose rasburicase in the treatment of adults with hyperuricemia associated with malignancy. Pharmacotherapy 2006;26:242-7. 